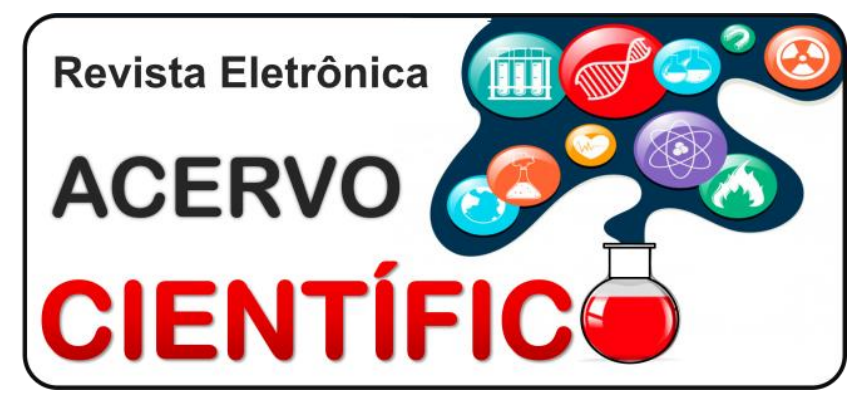

\section{REVISÃO BIBLIOGRÁFICA}

Recebido em: 12/2020

Aceito em: 1/2021

Publicado em: 1/2021

\title{
Fatores determinantes na caracterização da leptospirose como doença negligenciada: revisão integrativa
}

\author{
Determining factors in characterization of leptospirosis as a neglected disease: integrative \\ review
}

\section{Factores determinantes en la caracterización de la leptospirosis como enfermedad desconocida: revisión integrativa}

Francielly Baêta Lacerda ${ }^{1 *}$, Paula Schwenck Pereira ${ }^{1}$, Lúcia Meirelles Lobão Protti'.

\begin{abstract}
Resumo: As doenças negligenciadas englobam as patologias que estão presentes nas populações com poucos recursos financeiros. $\mathrm{O}$ artigo aborda sobre a leptospirose, sendo essa uma enfermidade infecciosa sistêmica com transmissão, principalmente, por meio do contato com a urina de roedores. $\mathrm{O}$ objetivo foi revisar aspectos da leptospirose que possam caracterizá-la como doença negligenciada. Para tanto foi realizada uma revisão integrativa através da busca de artigos nos bancos de dados da SciELO e PubMed, e coleta de informações secundárias, sendo dados obtidos através da plataforma do SINAN. A análise dos resultados demonstra que a leptospirose é uma doença prevalente no Brasil e a população mais acometida pela doença é aquela que possui uma baixa renda. Foram comparados os casos da leptospirose com a dengue (uma doença considerada como negligenciada) e verificado que apesar da dengue ter um maior número de caso, a leptospirose apresenta um maior número de óbitos. Em suma, a leptospirose deve ser incluída como uma doença negligenciada, pois de acordo com a Lei 13.930/19 o percentual dos recursos do Programa de Fomento à Pesquisa da Saúde deve ser aplicado para intervenções essas doenças, logo essa patologia necessita de uma maior visibilidade para ser reduzida na população.
\end{abstract}

Palavras-chave: Doenças negligenciadas, Leptospirose, Fatores sociais.

\begin{abstract}
Neglected diseases include pathologies that are present in populations with few financial resources. The article deals with leptospirosis, which is a systemic infectious disease with transmission, mainly through contact with rodent urine. The objective was to review aspects of leptospirosis that could characterize it as a neglected disease. For this purpose, an integrative review was carried out by searching for articles in the SciELO and PubMed databases, and collecting secondary information, with data obtained through the SINAN platform. The analysis of the results shows that leptospirosis is a prevalent disease in Brazil and the population most affected by the disease is the one with a low income. The cases of leptospirosis were compared with dengue (a disease considered as neglected) and found that despite the dengue has a higher number of cases, leptospirosis has a higher number of deaths. In short, leptospirosis must be included as a neglected disease, because according to Law 13,930 / 19 the percentage of resources from the Health Research Promotion Program must be applied for neglected disease interventions, so this pathology needs greater visibility to be reduced in the population.
\end{abstract}

Keywords: Neglected diseases, Leptospirosis, Social factors.

Resumen: Las enfermedades desatendidas incluyen patologías que están presentes en poblaciones con pocos recursos económicos. El artículo trata sobre la leptospirosis, que es una enfermedad infecciosa sistémica con transmisión, principalmente por contacto con orina de roedores. El objetivo fue revisar aspectos de la leptospirosis que pudieran caracterizarla como una enfermedad desatendida. Para ello, se

${ }^{1}$ Faculdade Dinâmica do Vale do Piranga (FADIP), Ponte Nova - MG. *E-mail: franciellybaeta@outlook.com 
realizó una revisión integradora mediante la búsqueda de artículos en las bases de datos SciELO y PubMed, y recolectando información secundaria, con datos obtenidos a través de la plataforma SINAN. El análisis de los resultados muestra que la leptospirosis es una enfermedad prevalente en Brasil y la población más afectada por la enfermedad es la de bajos ingresos. Los casos de leptospirosis se compararon con el dengue (una enfermedad considerada desatendida) y se encontró que a pesar de la el dengue tiene un mayor número de casos, la leptospirosis tiene un mayor número de muertes. En definitiva, la leptospirosis debe incluirse como una enfermedad desatendida, pues según la Ley $13.930 / 19$ el porcentaje de recursos del Programa de Promoción de la Investigación en Salud debe aplicarse para intervenciones de enfermedades desatendidas, por lo que esta patología necesita mayor visibilidad. reducirse en la población.

Palabras clave: Enfermedades desatendidas, Leptospirosis, Factores sociales.

\section{INTRODUÇÃO}

Algumas doenças são classificadas como negligenciadas, dentre essas patologias pode ser citado à esquistossomose, dengue e a malária. As doenças negligenciadas englobam as patologias que se encontram nas populações com poucos recursos financeiros ou em condições de pobreza e consequentemente auxiliam na continuidade do quadro de desigualdade social (MINISTÉRIO DA SAÚDE, 2010). Deve-se notar que, por se tratarem de doenças negligenciadas os métodos de tratamento e diagnósticos referentes a essas enfermidades necessitam de mais pesquisa e investimento para se tornarem efetivos (BBC, 2019).

Sabe-se que a leptospirose atinge um público mais carente, sendo essa uma doença infecciosa sistêmica, com a principal via de transmissão o contato direto ou indireto com a urina de animais, especialmente os ratos, atingindo tanto ambientes rurais quanto urbanos (BHARTI A, et al., 2003). A patologia apresenta uma evolução aguda, febril e é causada por uma bactéria do gênero Leptospira (FIGUEIREDO CM, et al., 2001). Em geral, ela se relaciona com as classes sociais e condições comportamentais, tendo um destaque maior em áreas mais pobres e com alto nível de desigualdade social. No Brasil, ela é considerada como uma enfermidade endêmica que se torna epidêmica nos períodos de chuva, tendo um maior destaque nas capitais e regiões metropolitanas, por causa das enchentes e da aglomeração de pessoas (FIOCRUZ, 2015).

É importante destacar que a leptospirose apresenta um amplo aspecto clínico, dessa forma existem diagnósticos diferenciais que podem atrasar a confirmação da doença. A leptospirose pode evoluir de várias formas, dependendo dos sintomas do paciente. Os médicos podem ter algumas hipóteses diagnósticas que cursam com o quadro da doença como, por exemplo, na fase precoce o paciente pode ter sintomas compatíveis com dengue, influenza, malária, doença de Chagas, entre outras. Já na fase tardia, os sintomas podem relacionar com dengue hemorrágica, hepatites virais agudas, pneumonias, pielonefrite, síndrome hepatorrenal, coledocolitíase esteatose aguda, entre outras (MINISTÉRIO DA SAÚDE, 2014).

Devido ao fato de a leptospirose atingir em maior escala as pessoas de baixa renda, essa enfermidade apresenta uma baixa visibilidade, como também a falta de auxílio do poder público para contribuir com a sua resolução (HOTEZ PJ, 2013). De acordo com o Sistema de Informação de Agravos de Notificação (SINAN) o Brasil teve 3.358 casos notificados de leptospirose em 2019. Portanto, é de extrema importância essa inserção de dados dos índices de leptospirose nas plataformas, para compreender se a mesma deve ser considerada uma doença negligenciada. Assim, objetivo foi revisar aspectos da leptospirose que possam caracterizá-la como uma doença negligenciada.

\section{MÉTODOS}

O estudo é caracterizado como uma revisão integrativa, com abordagem qualitativa. A busca dos artigos foi realizada de forma independente pelos pesquisadores nos bancos de dados Scientific Electronic Library Online (SciELO) e PubMed. A estratégia de busca foi fundamentada nos descritores do assunto e operadores booleanos "LEPTOSPIROSIS" AND "NEGLECTED DISEASES" OR "SOCIAL FACTORS". Os artigos selecionados foram escolhidos através de critérios de seleção pré-definidos entre os pesquisadores 
como: artigos que abordavam a epidemiologia da doença no Brasil, artigos sobre os sintomas e agravamentos da doença, trabalhos contendo dados da população acometida, pesquisas recentes publicadas entre os anos de 2000-2020. A seleção inicial foi realizada independentemente pelos pesquisadores, através do resumo de todos os artigos encontrados. Os artigos repetidos foram removidos verificando os autores do artigo, o título, o ano e o local de publicação. Os critérios de exclusão do estudo foram artigos duplicados, artigos que possuíam outra temática e estudos que abordavam a Leptospirose apenas em animas.

As buscas foram realizadas nas duas bases de dados e acrescentadas com informações secundárias, como de sites governamentais e livros. Além disso, para discussão e comparação com outras doenças já classificadas como negligenciadas, foram utilizados dados coletados na plataforma DATASUS, seguindo o caminho: SINAN (Sistema de Informação de Doenças e Agravados de Notificação), epidemiológicas e Morbidades. Os dados do SINAN foram confrontados com as informações obtidas através da revisão integrativa, utilizando o método de triangulação. De acordo com Santos et al. (2020), esse método permite a utilização de mais de uma técnica, teoria, fonte de dados ou investigador, possibilitando a apreensão de uma dada realidade sob diversos ângulos.

O fluxograma detalha o caminho seguido para busca dos resultados. Assim, foram utilizados 15 artigos associados às informações secundárias, provenientes de 6 fontes diferentes: Sistema de Informação de Agravos de Notificação (SINAN), Ministério da Saúde, Fundação Osvaldo Cruz (Fiocruz), British Broadcasting Corporation (BBC), World Health Organization (WHO) e o livro de Hotez PJ (2013) (Figura 1).

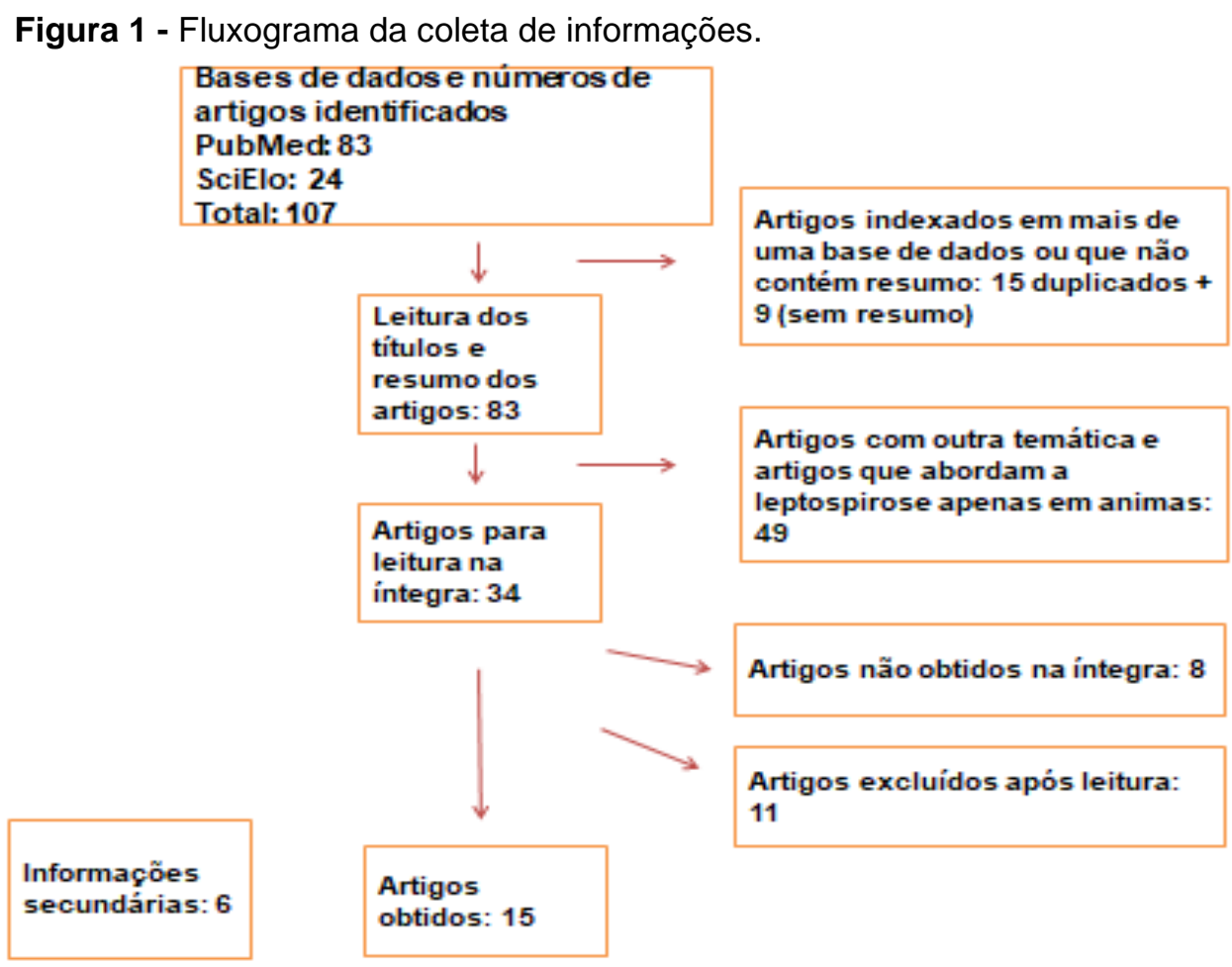

Nota: As fontes secundárias referem-se a sites governamentais e livros textos usados na complementação dos dados. Fonte: Lacerda FB, et al., 2021.

\section{RESULTADO E DISCUSSÃO}

O Quadro 1 traz o levantamento dos trabalhos utilizados para avaliação da leptospirose como doença negligenciada. A maior parte dos artigos considerados foram publicados após 2010, sendo 9 dos 15 utilizados no estudo. Entre as publicações 2 abordavam as dificuldades e importância de diagnóstico; 2 sobre os agravamentos da doença, 4 sobre a distribuição da doença na população, 6 sobre as doenças negligenciadas. 
Quadro 1 - Artigos usados na construção da revisão.

\begin{tabular}{|c|c|c|c|}
\hline Título & Objetivo principal & Autores/Ano & Principais resultados \\
\hline $\begin{array}{l}\text { Leptospira and leptospirosis. } \\
\text { microbiology }\end{array}$ & $\begin{array}{l}\text { Demonstrar a lepstospira } \\
\text { como fator determinante único } \\
\text { de virulência. }\end{array}$ & $\begin{array}{l}\text { ADLER B e } \\
\text { MOCTEZUMA ADLP, } \\
2010 .\end{array}$ & $\begin{array}{c}\text { Os antígenos de proteína recombinante são eficazes no } \\
\text { desenvolvimento de vacinas baseadas em antígenos protetores } \\
\text { definidos. }\end{array}$ \\
\hline $\begin{array}{l}\text { Validation of a case definition for } \\
\text { leptospirosis diagnosis in } \\
\text { patients with acute severe febrile } \\
\text { disease admitted in reference } \\
\text { hospitals at the State of } \\
\text { Pernambuco, Brazil }\end{array}$ & $\begin{array}{l}\text { Autenticar uma descrição de } \\
\text { caso através de dados clínicos } \\
\text { e laboratoriais, nos pacientes } \\
\text { hospitalizados com a hipótese } \\
\text { de leptospirose. }\end{array}$ & $\begin{array}{c}\text { ALBUQUERQUE F, et } \\
\text { al., } 2011 .\end{array}$ & $\begin{array}{c}\text { Foram estudados } 97 \text { pacientes, destes } 75 \text { com leptospirose e } 22 \\
\text { sem a doença. O número médio para confirmar o caso foi } \\
\text { contemplado. Apresentou sensibilidade de } 85,3 \% \text { e especificidade } \\
\text { de } 68,2 \% \text {, sendo essa combinação obtida por meio de } 7 \text { ou mais } \\
\text { critérios, obtendo valor preditivo positivo de } 90,1 \text { e negativa de } \\
57,7 \text {, tendo uma precisão de } 81,4 \% \text {. }\end{array}$ \\
\hline $\begin{array}{l}\text { Leptospirosis: a zoonotic } \\
\text { disease of global importance }\end{array}$ & $\begin{array}{l}\text { Demonstrar que a mortalidade } \\
\text { é significativa e está } \\
\text { relacionada tanto no atraso do } \\
\text { diagnóstico quanto à falta de } \\
\text { estrutura e suspeita clínica } \\
\text { precisa. }\end{array}$ & BHARTI A, et al., 2003. & $\begin{array}{c}\text { A sequência do genoma de Leptospira interrogans serovar lai e os } \\
\text { demais estudos sobre o sequenciamento do genoma da leptospira } \\
\text { prometem orientar os futuros estudos sobre a patologia. Ainda não } \\
\text { existe uma vacina disponível e a prevenção está relacionada com } \\
\text { as medidas de saneamento. }\end{array}$ \\
\hline $\begin{array}{l}\text { Knowledge, attitudes, and } \\
\text { practices related to leptospirosis } \\
\text { among urban slum residents in } \\
\text { Brazil. }\end{array}$ & $\begin{array}{l}\text { Abordar sobre } \\
\text { desproporcionalidade de como } \\
\text { a leptospirose afeta os } \\
\text { moradores de favelas } \\
\text { urbanas. }\end{array}$ & $\begin{array}{l}\text { DE ARAÚJO WN, et al., } \\
2013 .\end{array}$ & $\begin{array}{c}\text { Necessidade de tratamento focado em nível individual e familiar } \\
\text { para diminuir o risco da leptospirose, até que estabeleça } \\
\text { intervenções estruturais amplas para residentes de comunidades } \\
\text { marginalizadas. }\end{array}$ \\
\hline $\begin{array}{l}\text { Leptospirose humana no } \\
\text { município de Belo Horizonte, } \\
\text { Minas Gerais, Brasil: uma } \\
\text { abordagem geográfica. }\end{array}$ & $\begin{array}{c}\text { Apresentar dados da } \\
\text { leptospirose no município de } \\
\text { Belo Horizonte, Minas Gerais } \\
\text { e prover benefícios para as } \\
\text { próximas pesquisas e adoção } \\
\text { de medidas preventivas das } \\
\text { autoridades sanitárias de Belo } \\
\text { Horizonte. }\end{array}$ & $\begin{array}{l}\text { FIGUEIREDO CM, et } \\
\text { al., } 2001 .\end{array}$ & $\begin{array}{c}\text { Através da análise dos dados da leptospirose em Belo Horizonte } \\
\text { no ano de 1995, observou que dos } 30 \text { casos suspeitos e } 19 \\
\text { confirmados, havia predominância nas regiões norte, nordeste e } \\
\text { oeste, sendo que o foco da doença estava na favela. Foi notado } \\
\text { que ocorreu um aumento populacional na periferia e que nesta foi } \\
\text { encontrado a maioria dos casos confirmados e que este é o local } \\
\text { de maior carência de infraestrutura básica. }\end{array}$ \\
\hline
\end{tabular}




\begin{tabular}{|c|c|c|c|}
\hline Título & Objetivo principal & Autores/Ano & Principais resultados \\
\hline $\begin{array}{l}\text { One health: Endemic zoonoses } \\
\text { in the tropics: A public health } \\
\text { problem hiding in plain sight. }\end{array}$ & $\begin{array}{c}\text { Demonstrar que uma } \\
\text { abordagem sobre como } \\
\text { alcançar a saúde ideal para } \\
\text { pessoas, animais e o meio } \\
\text { ambiente é de extrema } \\
\text { importância para o combate } \\
\text { de doenças. }\end{array}$ & $\begin{array}{l}\text { HALLIDAY JEB, et al., } \\
2015 .\end{array}$ & $\begin{array}{l}\text { Necessário à implementação de medidas de prevenção e controle } \\
\text { que combatam essas doenças com estudos sobre a } \\
\text { implementação prática e avaliação de programas de controle de } \\
\text { doenças que reduzem a transmissão em populações animais e } \\
\text { consequentemente reduzem os causados sobre a saúde humana. }\end{array}$ \\
\hline $\begin{array}{l}\text { Brazil's neglected tropical } \\
\text { diseases: an overview and a } \\
\text { report card. }\end{array}$ & $\begin{array}{l}\text { Atualizar as últimas } \\
\text { informações sobre as doenças } \\
\text { tropicais negligenciadas } \\
\text { (DTNs) brasileiras. }\end{array}$ & $\begin{array}{c}\text { HOTEZ PJ e } \\
\text { FUJIWARA RT, } 2014 .\end{array}$ & $\begin{array}{c}\text { Foi observado que com algumas exceções, as DTNs brasileiras } \\
\text { continuam a ser difundidas entre os pobres e que essas doenças } \\
\text { precisam ser priorizadas por instituições acadêmicas e de } \\
\text { pesquisa do Brasil. }\end{array}$ \\
\hline $\begin{array}{l}\text { A leptospirose humana como } \\
\text { doença duplamente } \\
\text { negligenciada no Brasil. }\end{array}$ & $\begin{array}{l}\text { Investigar as discordâncias e } \\
\text { lacunas informacionais que } \\
\text { promovem uma versão } \\
\text { duplamente negligenciada da } \\
\text { leptospirose humana na } \\
\text { política pública de saúde } \\
\text { brasileira. }\end{array}$ & $\begin{array}{l}\text { MARTINS MHM, et al., } \\
2020 .\end{array}$ & $\begin{array}{c}\text { Constatou que essas discordâncias e lacunas informacionais } \\
\text { estão associadas ao fato de que a população acometida pela } \\
\text { leptospirose humana é uma população que o Estado não importa } \\
\text { em manter viva. }\end{array}$ \\
\hline $\begin{array}{c}\text { Doenças negligenciadas: } \\
\text { estratégias do Ministério da } \\
\text { Saúde. }\end{array}$ & $\begin{array}{l}\text { Apresentar as estratégias do } \\
\text { Ministério da Saúde sobre as } \\
\text { Doenças Negligenciadas. }\end{array}$ & $\begin{array}{l}\text { MINISTÉRIO DA } \\
\text { SAÚDE, } 2010 .\end{array}$ & $\begin{array}{c}\text { O aumento nos investimentos em doenças negligenciadas e nas } \\
\text { publicações nesta área mostra a necessidade de colocar em } \\
\text { pratica os conhecimentos sobre os assuntos perante a população } \\
\text { acometida, sendo elaborado um documento com o intuito de uma } \\
\text { criação eficaz do Programa Translacional do Brasil em Doenças } \\
\text { Negligenciadas. }\end{array}$ \\
\hline $\begin{array}{l}\text { Co-authorship network analysis: } \\
\text { a powerful tool for strategic } \\
\text { planning of research, } \\
\text { development and capacity } \\
\text { building programs on neglected } \\
\text { diseases. }\end{array}$ & $\begin{array}{l}\text { Desenvolver novos critérios e } \\
\text { abordagens por meio de } \\
\text { análise de redes sociais, e } \\
\text { com isso permitir uma } \\
\text { alocação justa e eficiente de } \\
\text { recursos sem prejuízo nos } \\
\text { padrões científicos. }\end{array}$ & $\begin{array}{l}\text { MOREL CM, et al., } \\
2009 .\end{array}$ & $\begin{array}{l}\text { Sobre o programa de doenças negligenciadas foi analisado a } \\
\text { produtividade científica de autores e instituições brasileiras em } \\
\text { periódicos internacionais com avaliação por pares. Expondo uma } \\
\text { grande variação entre as doenças cobertas pelo programa, } \\
\text { apresentando-se } 5 \text { vezes maior para a doença de Chagas e as } \\
\text { leishmanioses em relação com a dengue e a hanseníase. }\end{array}$ \\
\hline
\end{tabular}




\begin{tabular}{|c|c|c|c|}
\hline Título & Objetivo principal & Autores/Ano & Principais resultados \\
\hline $\begin{array}{l}\text { Improvement of leptospirosis } \\
\text { surveillance in remote Pacific } \\
\text { islands using serum spotted on } \\
\text { filter paper. }\end{array}$ & $\begin{array}{l}\text { Relatar sobre o uso de soro } \\
\text { manchado em papel de filtro } \\
\text { promovendo uma melhorar a } \\
\text { vigilância da leptospirose em } \\
\text { ambientes remotos e com } \\
\text { recursos limitados. }\end{array}$ & MUSSO D, et al., 2014. & $\begin{array}{l}\text { Entre os pacientes suspeitos de dengue, foram detectados } 5 \\
\text { infecções por leptospirose. }\end{array}$ \\
\hline $\begin{array}{c}\text { Optimal treatment of } \\
\text { leptospirosis: queries and } \\
\text { projections. }\end{array}$ & $\begin{array}{l}\text { Abordar importância do } \\
\text { tratamento ideal da } \\
\text { leptospirose. }\end{array}$ & $\begin{array}{l}\text { PAPPAS G e CASCIO } \\
\text { A, } 2006 .\end{array}$ & $\begin{array}{c}\text { Ainda existem dilemas antigos em relação ao tratamento da } \\
\text { leptospirose, informações novas experimentais ou clínica surge } \\
\text { poucas vezes persistindo ainda um entendimento inapropriado da } \\
\text { fisiologia da doença. }\end{array}$ \\
\hline $\begin{array}{c}\text { Revisão sistemática dos fatores } \\
\text { associados à leptospirose no } \\
\text { Brasil, 2000-2009. }\end{array}$ & $\begin{array}{l}\text { Revisar sobre os fatores } \\
\text { associados à leptospirose no } \\
\text { Brasil, durante 200-2009. }\end{array}$ & $\begin{array}{l}\text { PELISSARI DM, et al., } \\
\qquad 2011 .\end{array}$ & $\begin{array}{c}\text { Foi observado que } 7 \text { estudos relacionaram a ocorrência de chuva } \\
\text { ou enchentes com o crescimento do número de casos. A } \\
\text { leptospirose foi associada aos baixos níveis socioeconômicos, ao } \\
\text { aumento da precipitação pluviométrica e ao histórico familiar em } \\
\text { áreas urbanas, já em área rural, a doença foi vinculada às } \\
\text { atividades ocupacionais. }\end{array}$ \\
\hline $\begin{array}{l}\text { Overview of the epidemiology, } \\
\text { microbiology, and pathogenesis } \\
\text { of Leptospira spp. in humans. }\end{array}$ & $\begin{array}{l}\text { Abordar sobre a permanência } \\
\text { da subnotificação devido ao } \\
\text { grande espectro de sinais e } \\
\text { sintomas decorrente desse } \\
\text { patógeno e as suas } \\
\text { complicações. }\end{array}$ & $\begin{array}{l}\text { PLANK R e DEAN D, } \\
\qquad 2000 .\end{array}$ & $\begin{array}{c}\text { A leptospirose precisa ser considerada no diferencial diagnóstico } \\
\text { de paciente com doença febril aguda ou sintomas inespecíficos } \\
\text { que tem um diagnóstico epidemiológico compatível, sendo mais } \\
\text { frequente a infecção em zonas temperadas do que se imaginava } \\
\text { anteriormente, sendo necessárias mais pesquisas para a melhoria } \\
\text { no diagnóstico. }\end{array}$ \\
\hline $\begin{array}{l}\text { Leptospirosis: A Silent Epidemic } \\
\text { Disease. }\end{array}$ & $\begin{array}{l}\text { Abordar a leptospirose como } \\
\text { um todo, que atinge tanto a } \\
\text { saúde das pessoas, animais e } \\
\text { o meio ambiente. }\end{array}$ & $\begin{array}{l}\text { SCHNEIDER MC, et al., } \\
2013 .\end{array}$ & $\begin{array}{c}\text { É notório que uma abordagem geral dentro da interface animal- } \\
\text { humano-ecossistema é preciso para que possa compreender } \\
\text { sobre a previsão, detecção, prevenção e resposta a surtos de } \\
\text { leptospirose. }\end{array}$ \\
\hline
\end{tabular}

Fonte: Lacerda FB, et al., 2021. 
A Organização Mundial da Saúde (OMS) estima que mais de um bilhão de pessoas estão infectadas com pelo menos uma doença negligenciada e, apesar de potencialmente fatais, Halliday JEB, et al. (2015); corroborando com Hotez PJ (2013), afirmam que justamente por englobarem populações em condições precárias e não promoverem um retorno financeiro significativo, não despertam o interesse da indústria farmacêutica ou estudos de empresas privadas, tendo seus investimentos exclusivamente públicos.

As doenças negligenciadas são doenças transmitidas por agentes infecciosos que atingem desproporcionalmente as populações de baixa renda, que vivem em condições precárias de higiene, sem saneamento básico adequado ocorrendo principalmente na África, Ásia e América Latina (MINISTÉRIO DA SAUDE, 2010).

No Brasil, por meio de dados epidemiológicos e devido ao impacto das doenças negligenciadas, foram selecionadas algumas consideradas prioritárias para atuação sendo essas: dengue, doença de Chagas, esquistossomose, hanseníase, leishmaniose, malária, tuberculose (MINISTÉRIO DA SAÚDE, 2009).

Algumas doenças de acordo com World Health Organization (2020); são consideradas como doenças tropicais negligenciadas sendo essas: úlcera de Buruli, doença de Chagas, dengue, chicungunha, dracunculíase, equinococose, tripanossomíase africana, leishmaniose, hanseníase, filaríase linfática, micetoma, cromoblastomicose, oncocercíase, raiva, escabiose, esquistossomose, parasitoses, teníase, cisticercose, tracoma e bouba. Já as doenças negligenciadas consideradas prioritárias para a atuação no Brasil são: dengue, doenças de Chagas, esquistossomose, hanseníase, leishmaniose, malária e tuberculose.

No Brasil, o incentivo para as pesquisas sobre doenças negligenciadas deve ser direcionado pelo Ministério da Saúde que, através da Agência Nacional de Prioridades de Pesquisa em Saúde, define não só as oficinas que norteiam ações do Ministério da Educação, Ciência e Tecnologia, como também fundações de Amparo à Pesquisas e Secretarias (MINISTÉRIO DA SAÚDE, 2010).

A Iniciativa Medicamentos para doenças negligenciadas (Drugs for Neglected Diseases initiative - DNDi) é uma organização sem fins lucrativos, sua criação ocorreu no ano de 1999. No entanto, apenas no ano de 2003 seus trabalhos iniciaram de fato, após cinco instituições públicas se juntarem, com o objetivo de avaliar as condições dos pacientes, estabelecer parcerias e incentivos de pesquisas para os projetos, com objetivo de buscar novos recursos para os pacientes atingidos, a fim de melhorar a qualidade de vida, garantindo acesso universal e igualitário através de equidade, em que desde sua criação arrecadou muitos fundos, dentre esses 277 milhões foram dos recursos públicos e privados (MOREL CM, et al., 2009; MINISTÉRIO DA SAÚDE, 2018; MARTINS MHM, et al., 2020).

Através dos dados obtidos das oficinas, as pesquisas e ações se iniciaram no ano de 2003 pela tuberculose, e incluíram ao longo dos anos dengue e hanseníase. No ano de 2006 ocorreu a realização de definição de doenças prioritárias que engloba 7 doenças: dengue, doença de Chagas, esquistossomose, hanseníase, leishmaniose, malária, tuberculose. Além de implementação do Programa de Pesquisa e Desenvolvimento em doenças Negligenciadas no Brasil contando com diversos parceiros como a Secretária de Vigilância em Saúde e CNPq tendo como objetivo de destinar recursos financeiros para produção de inovação, focada em combate e tratamento das doenças, no qual, foram lançados editais e financiaram em media 140 projetos, investindo 39 milhões, além de investimentos também para outras doenças especificas (MOREL CM, et al., 2009; MINISTÉRIO DA SAÚDE, 2010).

A leptospirose é transmitida principalmente pelos roedores da espécie Rattus norvegicus (ratazana ou rato de esgoto) tal qual, não desenvolvem à doença, mas abrigam a bactéria nos rins e consequentemente eliminam no ambiente através da urina (MINISTÉRIO DA SAÚDE, 2014). O homem é hospedeiro terminal e acidental, o contágio ocorre por meio do contato com a urina dos roedores infectados (BHARTI A, et al., 2003) seja no solo, em alimentos ou na água (MINISTÉRIO DA SAÚDE, 2009) sendo necessária uma porta de entrada como escoriações na pele ou contato com as mucosas (HALLIDAY JEB, et al., 2015).

Conforme Hotez PJ e Fujiwara RT (2014), complementando Hotez PJ (2013), a transmissão está correlacionada a atividades ocupacionais e fatores ambientais, como períodos de chuvas e inundações, os 
quais ocorrem anualmente favorecendo o contato com as excretas, alcançando com mais facilidade populações que vivem em situações vulneráveis com falta de saneamento básico, educação em saúde, aglomerações e infestação de roedores.

O período de incubação varia de 5-14 dias, podendo estender até 30 dias, com os sintomas variando de olissintomáticos, à potencialmente graves (BHARTI A, et al., 2003; ADLER B e MOCTEZUMA ADLP, 2010). Segundo Halliday JEB, et al. (2015), além de Pelassari DM (2011), a fase precoce é responsável pela maioria dos casos, no entanto, o menor número de diagnósticos, devido as suas características, como autolimitação, inespecificidade dos sintomas e essencialmente pela dificuldade no diagnóstico (BHARTI A, et al., 2003; ALBURQUEQUE F, et al., 2011).

No final dessa fase são encontrados sinais característicos, que auxiliam na diferenciação do diagnóstico, como a sufusão conjuntival ocorrendo em $30 \%$ dos casos e intensa mialgia atingindo principalmente as panturrilhas (MINISTÉRIO DA SAÚDE, 2014). Nas manifestações clínicas da leptospirose o paciente pode apresentar no início da doença febre, cefaleia, mialgia, náuseas, vômitos, diarreia, artralgia, entre outros. Já nas formas graves uma das manifestações é a Síndrome de Weil. No entanto Hotez PJ e Fujiwara RT (2014); além de Plank R e Dean D (2000), afirmam que apesar da maioria dos pacientes evoluírem bem, $15 \%$ desenvolvem à fase tardia, com custos hospitalares elevados e principalmente a alta letalidade em média 50\%. Evidenciado pela Síndrome de Weil, manifestando à tríade clássica com Icterícia rubrica, Insuficiência Renal não oligúrica, com elevados índices da função renal e hipocalemicas, como também hemorragias, principalmente pulmonares, com sangramento maciço dentre esses 5 a $10 \%$ dos casos evolui de forma fatal em pouco tempo, inclusive com os recursos de terapêutico intensivo.

A leptospirose apresenta diversos registros no território brasileiro, sendo que a mesma apresenta uma maior incidência nas regiões sul e sudeste. Conforme os dados apontados pelo Sistema de Informação de Agravos de Notificação (SINAN) pode ser notado que no Brasil no ano de 2018 teve 88 casos confirmados de leptospirose sendo 9 desses no estado de Minas Gerais, porém esse número teve um elevado crescimento em 2019, apresentando 3358 casos, desses 172 são de Minas Gerais. Por outro lado, analisando também as doenças negligenciadas nos bancos de dados SINAN e buscando informações sobre o estado de Minas Gerais é possível observar que a esquistossomose apresentou 1.726 casos notificados no ano de 2017, já a dengue neste mesmo ano teve 25.949 casos e a malária apresentou em 202042 casos, todas essas enfermidades enquadradas no quesito de doenças negligenciadas, exceto a leptospirose.

Segundo Plank R e Dean D (2000), a leptospirose é uma zoonose febril aguda causada por bactérias do gênero Leptospira, uma espiroqueta aeróbica obrigatória de ampla distribuição mundial sendo à mais comum à da espécie Leptospira interrogans, apresentando estreita relação com a pobreza, atingindo principalmente a população economicamente desassistida (DE ARAUJO WN, et al., 2013; SCHNEIDER MC, et al., 2013; WORLD HEALTH ORGANIZATION, et al., 2011).

Dentre as doenças negligenciadas podemos fazer uma comparação entre a dengue e leptospirose observando os investimentos feitos, os números de casos e números de óbitos, em relação aos gastos hospitalares no Brasil entre 2003 a 2018 foram investidos $R \$ 270.739 .122,53$ para dengue, em contrapartida para a leptospirose o recurso foi muito menor, $\mathrm{R} \$ 30.341 .984,22$. De acordo com os dados do SINAN é possível observar entre de casos e óbitos das duas enfermidades e perceber que apesar da leptospirose apresentar uma quantidade menor de casos tem maior óbito em relação aos anos descritos. Nos anos de 2011 a 2016 a dengue teve 6.584 .442 casos resultando em 923 óbitos, já a leptospirose neste mesmo período apresentou 24.289 casos e 1980 mortes.

Fazendo uma análise do perfil populacional da leptospirose e comprando com o perfil da dengue é notável algumas diferenças, de acordo o SINAN nos anos de 2007 a 2012 são apresentados 4 fatores relacionados as doenças, sendo esses fatores: sexo, idade, escolaridade e raça. A leptospirose é predominante em homens $(78,60 \%$ homens, $21,30 \%$ mulheres), enquanto a dengue apresenta pouca diferença entre os sexos (feminino $44,80 \%$, masculino $55,10 \%$ ), já no quesito raça a leptospirose predomina na raça preta (branco $46 \%$, preto/pardo $41,20 \%$, amarelo $0,50 \%$, indígena $0,30 \%$ ), comparado com a 
dengue (branco 28,50\%, preto/pardo 35,40\%, amarelo 0,90\%, indígena 0,30\%), essa apresenta uma porcentagem menor nessa raça. A dengue apresenta uma porcentagem menor em pessoas de baixa escolaridade do que a leptospirose (Nono ano incompleto: dengue apresenta 18,50\% e a leptospirose 18,50\%). Em relação a idade entre 20 a 59 anos a leptospirose acomete mais pessoas comparado a dengue (leptospirose 72,30\%, dengue 60,60\%).

Para o diagnóstico enfrenta diversas dificuldades, pois a fase precoce engloba sintomas como febre de início súbito, cefaleia, mialgia e anorexia, sendo necessária a realização de diferenciação de diagnósticos com Dengue, Influenza, Malária, Riquetsioses, Doença de Chagas aguda, entre outras, realizado através de exames laboratoriais englobando: Hemograma completo, avaliação da função renal, função hepática, sódio e potássio (ALBURQUEQUE F, et al., 2011; MINISTERIO DA SAÚDE, 2014; PELISSARI DM, et al., 2011).

Entretanto é necessário um diagnóstico definitivo identificado através do isolamento da Leptospira, dispondo, como alternativa os métodos sorológicos como ELISA-IgM que é especifico e sensível, porém permite um diagnostico apenas retrospectivo, uma vez que os anticorpos só aparecem 7-10 dias. Outra opção é reação em cadeia de polimerase (PCR) é um exame sensível e com diagnóstico precoce, mas, tem custo elevado. Com isso, o método mais utilizado é teste de aglutinação microscópica (MAT), sendo necessário a coleta de 2 amostras de sangue com 2 semanas de diferença, considerando positivo quando os valores estão quatro vezes maiores, sendo, portanto, um diagnostico tardio (MINISTÉRIO DA SAÚDE 2014; ALBURQUEQUE F, et al., 2011).

Dessa forma, de acordo com Alburqueque F, et al. (2011), Pelassari DM, et al. (2011), a leptospirose é uma doença de difícil, tardio e desatualizado diagnostico, ocasionando além de subdiagnósticos, subnotificações e consequentemente não gerando a visibilidade necessária para investigações ambientais e epidemiológicas da Vigilância Sanitária em saúde, que poderiam reduzir a população expostas e os agravos em saúde (WORLD HEALTH ORGANIZATION, et al., 2010; MUSSO D, et al., 2014).

O tratamento é baseado em antibioticoterapia, no entanto sua eficácia é maior quando introduzido precocemente. Na fase inicial as opções de antibióticos são Amoxicilina: $500 \mathrm{mg}, \mathrm{VO}, 8 / 8 \mathrm{~h}$, por 5 a 7 dias, como opção Doxiciclina $100 \mathrm{mg}$, VO, 12/12h, por 5 a 7 dias e nos casos de contraindicação podemos incluir como alternativa Azitromicina ou Claritromicina. Já na fase tardia o medicamento de escolha é Penicilina $G$ Cristalina: 1.5 milhões UI, IV, de 6/6 horas; ou Ampicilina: $1 \mathrm{~g}$, IV, 6/6h; ou - Ceftriaxona: 1 a 2 g, IV, 24/24h ou Cefotaxima: $1 \mathrm{~g}$, IV, 6/6h. Alternativa: Azitromicina $500 \mathrm{mg}$, IV, 24/24h, todos utilizados por IV durante no mínimo 7 dias. Além disso, são importantes as medidas de suporte com sintomáticos, evitando aspirinas, como também hidratação venosa para reposição volêmica, exames para testes específicos e retornos de 24-72horas, nos casos mais graves não ocorra diurese espontânea deve lançar mão do uso de furosemida, caso sem melhora uma opção é hemodiálise de indicação precoce, além de observação dos eletrólitos como potássio. Para os contactantes é indicado quimioprofilaxia pós- exposição utilizando Doxiclina $100 \mathrm{mg}$ VO 12/12h por 5- 7dias (MINISTERIO DA SAUDE, 2018; PAPPAS G e CASCIO A, 2006).

A leptospirose é uma doença prevalente no Brasil e afeta profundamente a qualidade de vida dos contaminados, além de gerar impactos socioeconômicos negativos na população. Sabendo que as populações mais afetadas por essa doença são de baixa renda, essa patologia deve ser considerada como negligenciada, visto que a Lei 13.930/19 garante que parte dos recursos do Programa de Fomento à Pesquisa em Saúde seja destinada para as doenças negligenciadas ou raras, além disso, Brasil (2014); institui a Rede Nacional de Pesquisa em Doenças Negligenciadas (RNPDN) com o intuito de desenvolver pesquisas nessa área, dessa forma, aumentaria a responsabilidade do governo em promover politicas preventivas em relação a essa doença, além de contribuições por partes de intuições, como a DNDi, para um aumento na pesquisa e investimento não só no tratamento, mas também diagnóstico da doença.

\section{CONSIDERAÇÕES FINAIS}

Em suma, é possível perceber que a leptospirose apresenta um elevado impacto na saúde e o perfil da população acometida gera uma falta de interesse no âmbito público. Para chegar a essa conclusão foi exposto no artigo os fatores relacionados à leptospirose comparando os mesmo com a dengue, com isso 
pode-se observar que as pessoas acometidas pela leptospirose em grande maioria são pessoas com baixa renda, já a dengue acomete um grupo mais amplo. Dessa forma, ficando nítida a indiferença com a leptospirose e que é preciso uma maior visibilidade e recursos para essa doença.

\section{REFERÊNCIAS}

1. ADLER B. MOCTEZUMA ADLP. Leptospira and leptospirosis. Veterinary microbiology, 2010; 140(3-4): $287-296$.

2. ALBUQUERQUE F, et al. Validation of a case definition for leptospirosis diagnosis in patients with acute severe febrile disease admitted in reference hospitals at the State of Pernambuco, Brazil. Revista da Sociedade Brasileira de Medicina Tropical, 2011; 44(6): 735-739.

3. BBC. As doenças negligenciadas pela indústria farmacêutica que afetam milhões de pessoas no mundo e no Brasil. Disponível em: https://www.bbc.com/portuguese/geral-46961306. Acesso em: 28 fev. 2020.

4. BHARTI A, et al. Leptospirosis: a zoonotic disease of global importance. The Lancet infectious diseases, 2003; 3(12): 757-771.

5. BRASIL. Ministério da Saúde. Gabinete do Ministro. Portaria n 191, de 31 de Janeiro de 2014. Brasília, 2014. Disponível em: http://bvsms.saude.gov.br/bvs/saudelegis/gm/2014/prt0191_31_01_2014.html. Acesso em: 15 jun. 2020

6. DE ARAÚJO WN, et al. Knowledge, attitudes, and practices related to leptospirosis among urban slum residents in Brazil. The American journal of tropical medicine and hygiene, 2013; 88 (2): 359-363.

7. FIGUEIREDO CM, et al. Leptospirose humana no município de Belo Horizonte, Minas Gerais, Brasil: uma abordagem geográfica. Revista da sociedade brasileira de medicina tropical, 2001; 34(4): 331-338.

8. FIOCRUZ. Leptospirose. Disponível em: https://agencia.fiocruz.br/leptospirose-0. Acesso em: 20 mar. 2020.

9. HALLIDAY JEB, et al. One health: Endemic zoonoses in the tropics: A public health problem hiding in plain sight. The Veterinary Record, 2015; 176(9): 220-2015.

10. HOTEZ PJ. Forgotten people, forgotten diseases: the neglected tropical diseases and their impact on global health and development. John Wiley \& Sons, 2013.

11. HOTEZ PJ, FUJIWARA RT. Brazil's neglected tropical diseases: an overview and a report card. Microbes and infection, 2014; 16(8): 601-606.

12. MARTINS MHM, et al. A leptospirose humana como doença duplamente negligenciada no Brasil. Ciência \& Saúde Coletiva, 2020; 25: 919-928.

13. MINISTÉRIO DA SAÚDE. Doenças negligenciadas: estratégias do Ministério da Saúde. Revista Saúde Pública, 2010; 44(1): 200-2.

14. MINISTÉRIO DA SAÚDE. Guia de Vigilância em saúde. Brasília - DF, 2014; 2: 569-588.

15. MINISTÉRIO DA SAÚDE. Leptospirose diagnóstico e manejo clinico. Disponível em: https://bvsms.saude.gov.br/bvs/publicacoes/leptospirose-diagnostico-manejo-clinico2.pdf. Acesso em: 20 abr. 2020.

16. MINISTÉRIO DA SAÚDE. Saúde Brasil 2017: Uma análise da situação de saúde e os desafios para o alcance dos Objetivos de Desenvolvimento Sustentável. Brasília - DF, 2018; 1: 99-142.

17. MOREL CM, et al. Co-authorship network analysis: a powerful tool for strategic planning of research, development and capacity building programs on neglected diseases. PLoS neglected tropical diseases, 2009; 3(8).

18. MUSSO D, et al. Improvement of leptospirosis surveillance in remote Pacific islands using serum spotted on filter paper. International Journal of Infectious Diseases, 2014; 20: 74-76.

19. PAPPAS G, CASCIO A. Optimal treatment of leptospirosis: queries and projections. International journal of antimicrobial agents, 2006; 28(6): 491-496.

20. PELISSARI DM, et al. Revisão sistemática dos fatores associados à leptospirose no Brasil, 20002009. Epidemiologia e Serviços de Saúde, 2011; 20(4): 565-574.

21. PLANK R, DEAN D. Overview of the epidemiology, microbiology, and pathogenesis of Leptospira spp. in humans. Microbes and infection, 2000; 2(10): 1265-1276.

22. SCHNEIDER MC, et al. Leptospirosis: A Silent Epidemic Disease. International journal of environmental research and public health, 2013; 10(12): 7229-7234.

23. SISTEMA DE INFORMAÇÃO DE AGRAVOS E NOTIFICAÇÃO. Leptospirose. Disponível em: https://portalsinan.saude.gov.br/leptospirose. Acesso em: 20 abr. 2020.

24. WORLD HEALTH ORGANIZATION. 2020. In: Control of Neglected Tropical Diseases. Disponível em: https://www.who.int/teams/control-of-neglected-tropical-diseases. Acesso em 02 set. 2020.

25. WORLD HEALTH ORGANIZATION, et al. Report of the first meeting of the leptospirosis burden epidemiology reference group. World Health Organization, 2010. Disponível em: https://apps.who.int/iris/bitstream/handle/10665/44382/9789241599894_eng.pdf. Acesso em: 02 abr. 2020.

26. WORLD HEALTH ORGANIZATION, et al. Report of the second meeting of the leptospirosis burden epidemiology reference group. World Heath Organization, 2011. Disponível em: https://apps.who.int/iris/bitstream/handle/10665/44588/9789241501521_eng.pdf?sequence=1. Acesso em 02 abr. 2020. 